\title{
Analysis of Wavelet-based Transform Compression Techniques on Medical Image
}

\author{
A. O. Ajao \\ Department of Computer Engineering, \\ The Federal Polytechnic, Offa, Nigeria
}

\author{
T. S. Ibiyemi \\ Department of Electrical and Electronics Engineering, \\ University of Ilorin, Ilorin, Nigeria
}

\begin{abstract}
This paper is aimed at analyzing the performance of three different state-of-the-art image compression schemes namely Embedded Zerotree Wavelet (EZW), Spatial-orientation Trees Wavelet (STW) and Set Partitioning in Hierarchical Trees (SPHIT). The paper analyses the compression schemes using $\mathrm{X}$-ray image data such that the quality of the reconstructed image would be closely related to its original image after 20 iterations of the compression steps. We compared the compression ratio and bit per pixel against the peak signal to noise ratio respectively for X-ray images represented in various image sizes of 256x256 and 512×512. Also, we discussed the characteristics on quality performance used. Some tests conducted for comparing them and the compression quality are addressed in this paper and the quality of compression is determined from the metrics of compression ratio (CR), bit per pixel (BPP), mean square error (MSE) and peak signal to noise ratio (PSNR).
\end{abstract}

\section{General Terms}

Image Process, Wavelet Analysis, Image Compression.

\section{Keywords}

Compression, X-ray Images, Discreet Wavelet Transform, Bitrate.

\section{INTRODUCTION}

Image compression $[1,2,3]$ is one of the key aspects in image processing applications. An effectively high compression scheme for reconstructing X-ray images without apparent human perceptual degradation or loss of fidelity is one of the key requirements to fully leverage low bit-rate and high throughput transmission and storage of medical images $[4,5]$. Image compression is an essential steps towards alleviating the problems of large demands for image data storage and transmission capability, which is a major challenge for applications related to Biomedical, Telemedicine, Teleconsultation, Teleradiology and Telecommunication.

In the last two decades, there has been tremendous growth in the field of wavelet-based compression, which has also lead to the worldwide standard compression and coding scheme by Joint Photographic Experts Group (JPEG2000) for image storage and transmission [6]. The key challenges regarding image compression is finding the best compromise between a low compression ratio and a good perceptual result [7]. Hence determining a simple and effective compression scheme related to the quality of reconstructed image becomes essential to the medical service providers.

This paper uses three wavelet based techniques to compress and reconstruct X-ray images. These include Embedded Zero Tree Wavelet (EZW), Set Partitioning in Hierarchical Trees
(SPIHT) and Spatial-orientation Tree Wavelet (STW). The comparative analysis is introduced to determine the technique with the best performance in terms of compression ratio (CR), bit per pixel (BPP), mean square error (MSE) and peak signal to noise ratio (PSNR).

\section{IMAGE COMPRESSION SCHEME}

The increasing growth of medical image modalities is expected to give rise to applications of compression schemes with an agreed quality assurance of the medical images. The requirements of the compression scheme for the medical image data are robustness and speed of compression and reconstruction. The essential point is that assessment scheme should highlight storage capacity and data transmission rate that may affect the storage capacity and spectrum availability issue of transmission channels [8].

In image compression and reconstruction, a number of different mathematical transforms are used according to their suitability for different methods which are classified as lossless or lossy compression methods. In lossless compression, the original representation is recovered perfectly while in lossy compression certain degradation may be observed. The visual quality of compressed medical images is imperative because of the fact that degradation in medical images is unacceptable in medical practices [9]. The application of digital image compression needs to be carefully selected according to the characteristics of the image of interests in order to meet the following requirements as stated in $[10,11]$.

- Better performances than existing standards for strong compression ratios devoid of degradation,

- A progressive transmission of compressed images with increasing quality of achieving lossless compression,

- Low computation cost and complexity with ease of hardware implementation of the algorithm,

- Good data security and robustness with respect to errors during transmission.

\subsection{Wavelet Theory}

Wavelets $[12,13]$ are basically functions that are localized in frequency around a central value and are limited in time i.e. they are of finite support and hence localized in time around a central value. Wavelets exhibit constant shape because they are generated from only one function called the mother wavelet. The continuous wavelet transform (WT) is defined by $[14,15]$ :

$C W T(a, b)=\frac{1}{\sqrt{a}} \int_{-\infty}^{\infty} f(t) \psi\left(\frac{t-b}{a}\right) d t$

where $a \in \mathfrak{R}^{+}-\{0\}$ is a scale parameter, $b=\mathfrak{R}$ is a shift parameter 
The basic wavelet generates a set of wavelet basis functions:

$$
\psi_{a, b}(t)=\frac{1}{\sqrt{a}} \psi\left(\frac{t-b}{a}\right)
$$

Since discrete wavelet transform (DWT) is a sampled version of CWT, the DWT is defined by:

$$
\begin{aligned}
& D W T(a, b)=\sum s(n) \psi_{j, k}(n) \\
& \text { where } a=2^{-j}, \quad b=k 2^{-j}, \quad j \in N, \quad k \in Z \\
& \psi_{j, k}(t)=\frac{1}{\sqrt{2^{-j}}} \psi\left(\frac{t}{2^{-j}}-k\right)=2^{\frac{j}{2}} \psi\left(2^{j} t-k\right)
\end{aligned}
$$

In the following section the compression methods used in the evaluation are briefly explained.

\subsubsection{Spatial-orientation Tree Wavelet (STW) Coding}

The Spatial Orientation Trees are techniques of partitioning wavelet trees in a manner that tends to keep insignificant coefficients together in larger subsets. It is designed to exploit the spatial relationships between the wavelet coefficients in the different levels of the subband pyramid by using a state transition model which allows it to reduce the number of bits needed for encoding. Further description of the STW coding algorithm can be found in [16]

\subsubsection{Embedded Zero-Tree Wavelet (EZW) Coding} This Wavelet coding technique was introduced by Shapiro [17] as a quantization and coding strategy based on discrete wavelet transform characteristics for image compression. It is designed to attain an optimal representation of coding positions by using significant coefficients in one frequency band to predict the location and value of significant coefficients in other bands. The term "embedded" is a process of encoding the transform magnitudes that allows for progressive transmission of the compressed image. While, the "Zerotrees" refers to the concept that allows for a concise encoding of the positions of significant values that is obtained during the embedded coding process. The algorithm forms a hierarchical quad tree data structure for the wavelet-transformed coefficients. A detailed description of the EZW coding algorithm is given in [17].

\subsubsection{Set Partitioning in Hierarchical Trees (SPIHT) coding}

This coding scheme proposed by Said and Pearlman [18] is an algorithm in the subband/wavelet/pyramid coding class. The scheme is designed to group coefficients of comparable significance across scales by spatial location in bands that are oriented in the same direction thereby compressing bit stream in which the most important coefficients are transmitted first. These codes typically decompose the image using an octave subband, critically sampled pyramid, or complete wavelet transformation, and then code the resulting transform coefficients in an efficient way. The most efficient wavelet coding techniques exploit both the spatial and frequency localization of wavelets. The reader is referred to reference [18] for a fuller discussion and details of implementation.

\section{WAVELET CODING TECHNIQUES}

The generic steps of Wavelet Coding Techniques are shown in Figure 1 as follows:

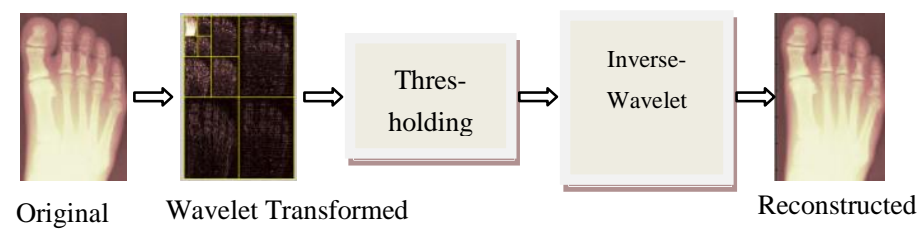

Figure 1. Generic block diagram of a transform compression scheme [19]

\section{Step 1: Image decomposition}

An image transformation is set to obtain the image characteristics so as to accomplish the compression task as shown in Figure 1. The image is decomposed into subbands, such that lower subbands correspond to higher image frequencies and higher subbands correspond to lower image frequencies, where most of the image energy is concentrated. The decomposition is typically produced by an analysis filter bank followed by downsampling.

A given image $\mathrm{f}(x, y)$ of size $(M \times N)$ is decomposed using Fast Wavelet transform following the equations (5)-(10) found in [20]:

The forward Discrete Wavelet transform of a digital image $f(x, y)$ of size $M x N$ is defined by:

$$
\begin{aligned}
& W_{\phi}\left(j_{0}, m, n\right)=\frac{1}{\sqrt{M N}} \sum_{x=0}^{M-1} \sum_{y=0}^{N-1} f(x, y) \phi_{j_{0}, m, n}(x, y) \\
& W_{\psi}^{i}(j, m, n)=\frac{1}{\sqrt{M N}} \sum_{x=0}^{M-1} \sum_{y=0}^{N-1} f(x, y) \psi_{j_{, m, n}}^{i}(x, y)
\end{aligned}
$$

where

$i=\{$ Horizontal $(h), \operatorname{Vertical}(v)$, Diagonal $(d)\}$ representations.

The 2D scaling function Equation (5) represent a twodimensional scaling function, $\varphi(x, y)$ while Equation (6) represent a three-dimensional wavelets $\psi^{v}(x, y), \psi^{h}(x, y)$, and $\psi^{d}(x, y)$, which measure variation along vertical direction, horizontal direction and diagonal direction respectively as shown in Equations (7)-(10).

$$
\begin{array}{ll}
\varphi(x, y)=\varphi(x) \times \varphi(y) & ; \text { scaling function } \\
\psi^{v}(x, y)=\varphi(x) \times \psi(y) & ; \text { vertical multiresolution } \\
\psi^{h}(x, y)=\psi(x) \times \varphi(y) & ; \text { hrizontal multiresolution } \\
\psi^{d}(x, y)=\psi(x) \times \psi(y) & ; \text { diagonal multiresolution }
\end{array}
$$

\section{Step 2: Image Thresholding}

The widely used thresholding technique for signal and image compression as found in $[21,22]$ are discussed as follows:

- Hard Thresholding. This is defined as:

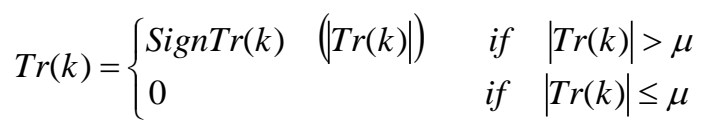

where $\mu$ is the threshold. Equation (11) implies that the coefficients that are above the threshold are selected while the coefficients whose absolute values are lower than the threshold are set to zero. 
- Soft Thresholding. The general soft shrinkage rule is defined by:

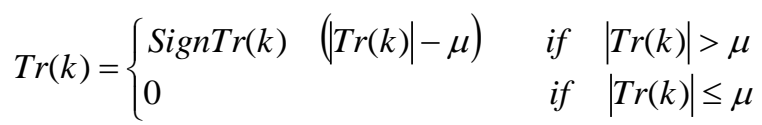

This removes the coefficients below the threshold and scales the ones that are left.

\section{Step 3: Image Reconstruction}

The inverse Wavelet transform is derived for $\mathrm{f}(\mathrm{x}, \mathrm{y})$ as follows:

$$
\begin{aligned}
& f(x, y)=\frac{1}{\sqrt{M N}} \sum_{m} \sum_{n} W_{\phi}\left(j_{0}, m, n\right) \phi_{j_{0}, m, n}(x, y)+ \\
& \frac{1}{\sqrt{M N}} \sum_{i=H, V, D} \sum_{j=j_{0}}^{\infty} \sum_{m} \sum_{n} W_{\psi}^{i}(j, m, n) \psi_{j, m, n}^{i}(x, y)
\end{aligned}
$$

\subsection{Image Quality Evaluation}

The quality of the image signal can be measured using different models. The commonly used metrics to evaluate the perceptual quality of reconstructed image are [23, 24]:

- The Mean Square Error (MSE). It represents the mean squared error between the compressed and the original image and is given by:

$M S E=\frac{1}{N \times M} \sum_{i=0}^{N-1} \sum_{j=0}^{M-1}\left(x_{i, j}-y_{i, j}\right)^{2}$

where $x_{i, j}$ and $y_{i, j}$ represent the original image and the reconstructed image respectively. The lower value of MSE corresponds to lower error in the reconstructed image.

- The Peak Signal to Noise Ratio (PSNR). This represents a measure of the peak error and is expressed in decibels. It is defined by:

$$
\begin{gathered}
P S N R=10 \times \log _{10}\left\{\frac{255^{2}}{M S E}\right\} \text { or } \\
20 \times \log _{10}\left\{\frac{255}{\sqrt{M S E}}\right\}
\end{gathered}
$$

where

MSE is mean squared error between the original and the reconstructed image. In this metric, higher PSNR denotes better quality of the compressed or reconstructed image.

The compression ratio. This signifies that the compressed image is stored using only $\mathrm{CR} \%$ of the initial storage size and is given by the following:

$$
C R=\frac{\text { Uncompress size }}{\text { Compress size }}
$$

Also, It can be represented in terms of percentage space saving (PSS) given by:

$$
P S S=1-\frac{1}{C R}
$$

\section{RESULTS AND DISCUSSION}

We carried out a comparative study of wavelet-based compression schemes for still medical images. The compression schemes are performed by using the Embedded Zerotree Wavelet (EZW), Spatial-orientation Trees Wavelet (STW) and Set Partitioning in Hierarchical Trees (SPHIT) algorithms in the MATLAB toolbox. The algorithm is tested on X-ray image of size $256 \times 256$ and $512 \times 512$ and each was performed for 20 different iteration levels. Figure 2 and Figure 3 show the plots which were obtained from SPIHT, EZW and STW during the process, for X-ray image of size $256 \times 256$ and $512 \times 512$ respectively.

The plots at significant levels of compression ratios (Figures 2 and 3) indicates that the PSNR between the original and reconstructed images were significantly higher for the X-ray images used than for the other two algorithms. The results for the CR against PSNR however closely follow the same pattern.

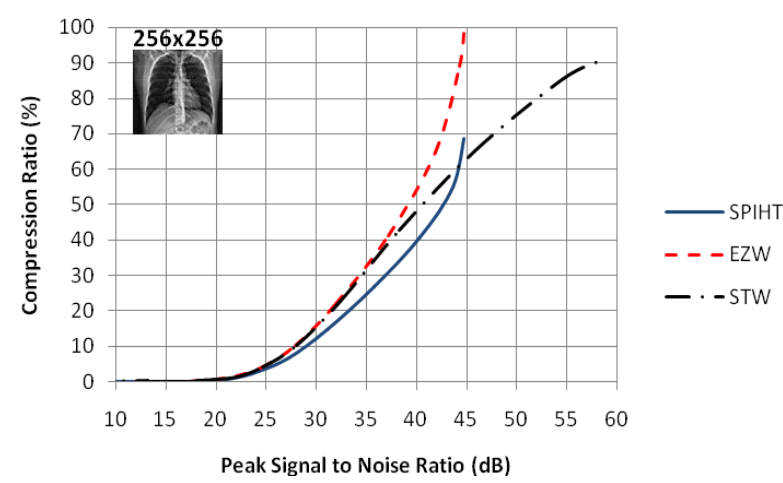

Figure 2. The plot of CR versus PSNR for 256x256 image by applying the three compression schemes.

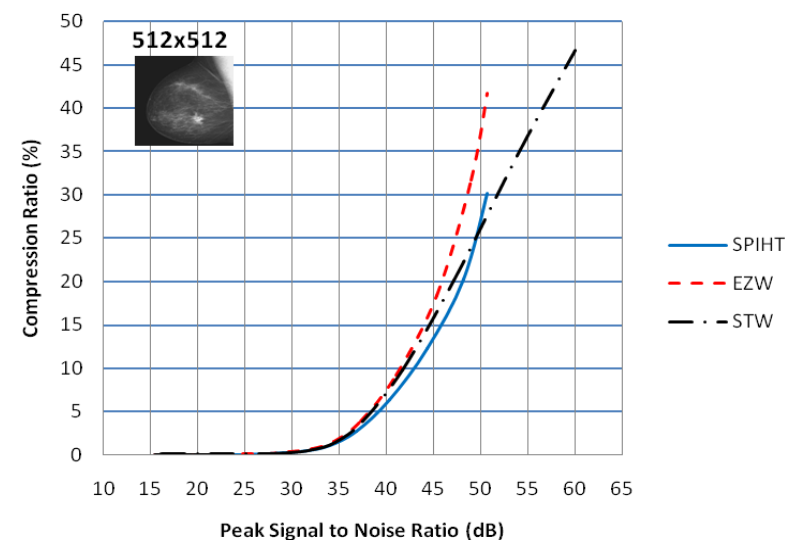

Figure 3. The plot of CR versus PSNR for $512 \times 512$ image by applying the three compression schemes.

Results are observed in terms of compression ratio (CR), bit per pixel (BPP), mean square error (MSE) and peak signal to noise ratio (PSNR). Notice that the performance of coding in PSNR value (in $\mathrm{dB}$ ) increases when the MSE decreases. Similar experimental results are obtained for the various X-ray image resolutions.

The simulation results in Figures 4 - 6 show the visual quality of full image reconstruction and their performance gain at 
iteration level 10 and 15 for various wavelet based compression scheme.
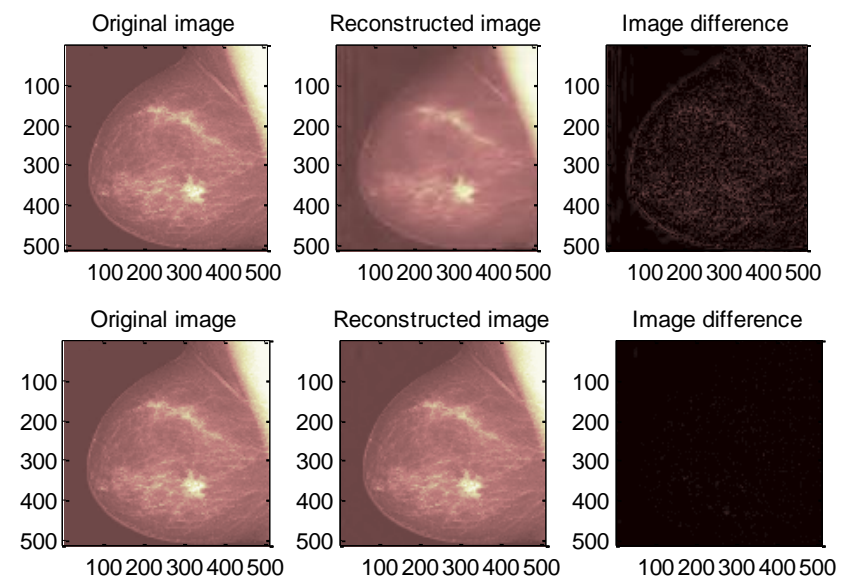

Figure 4. SPIHT: (Top $=10$ steps $) \quad \mathrm{CR}=0.46 \%, \quad \mathrm{BPP}=0.04$ $\mathrm{MSE}=44.76$ and $\mathrm{PSNR}=31.62$ (Bottom $=15$ steps) $\mathrm{CR}=20.28 \%$, $\mathrm{BPP}=1.62, \mathrm{MSE}=0.98$ and $\mathrm{PSNR}=48.21$
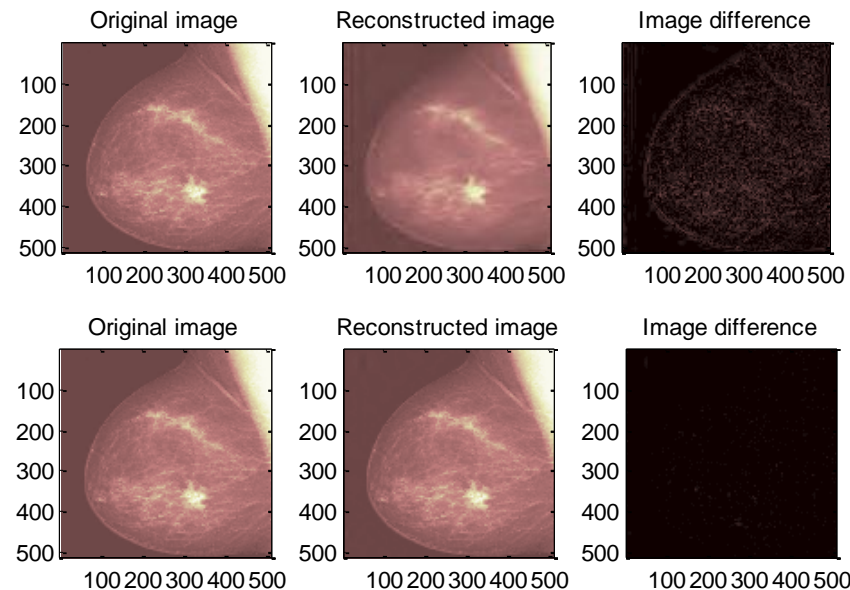

Figure 5. EZW: $(\mathrm{Top}=10 \mathrm{steps}) \mathrm{CR}=0.66 \%, \mathrm{BPP}=0.05$, MSE $=39.59$ and PSNR=32.16 (Bottom=15steps) $\mathrm{CR}=31.68 \%, \mathrm{BPP}=2.53, \mathrm{MSE}=\mathbf{0 . 8 2}$ and $\mathrm{PSNR}=48.98$
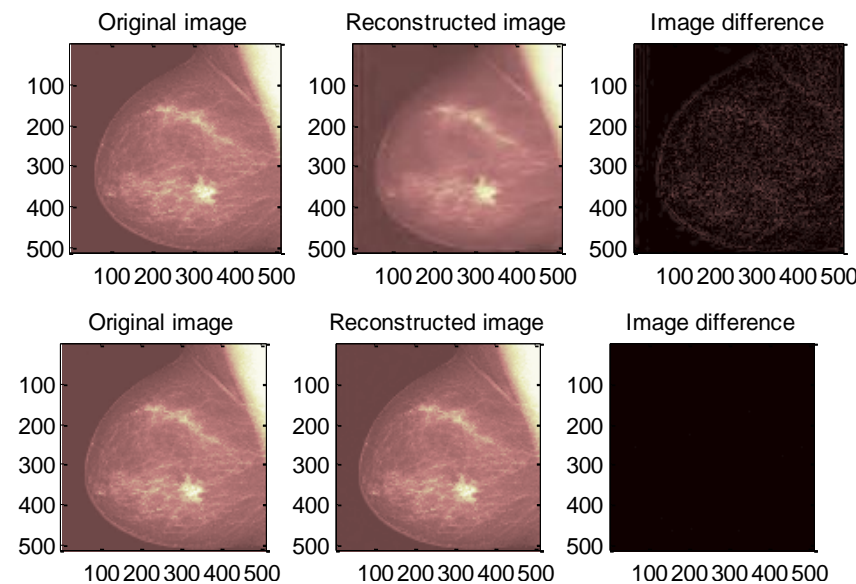

Figure 6. STW: $($ Top $=10 \mathrm{steps}) \mathrm{CR}=\mathbf{0 . 5 6 \%}$, BPP=0.04, MSE $=39.33$ and PSNR=32.18 $($ Bottom $=15$ steps $)$

$\mathrm{CR}=35.18 \%, \mathrm{BPP}=2.81, \mathrm{MSE}=\mathbf{0 . 2 5}$ and $\mathrm{PSNR}=54.21$

It is observed that the performance gain for iteration level 17 through 20 is meaningless, because all the evaluation metrics have reached the maximum value and the perceptual quality of reconstructed image produce almost the same thing as shown in the Table 1, 2, 3 for SPIHT, EZW and STW respectively.

Table 1. The qualitative evaluation of images at different iteration level for SPIHT

\begin{tabular}{|l|l|l|l|l|}
\hline \multicolumn{5}{|l}{$\begin{array}{l}\text { SPIHT } \\
\text { Time of execution is } \mathbf{7 4 . 4 1 2 5 s}\end{array}$} \\
\hline Steps & CR & BPP & MSE & PSNR \\
\hline 10 & 0.46 & 0.04 & 44.76 & 31.62 \\
\hline 11 & 1.10 & 0.09 & 25.92 & 33.99 \\
\hline 12 & 2.73 & 0.22 & 13.58 & 36.80 \\
\hline 13 & 6.35 & 0.51 & 6.06 & 40.31 \\
\hline 14 & 12.22 & 0.98 & 2.40 & 44.32 \\
\hline 15 & 20.28 & 1.62 & 0.98 & 48.21 \\
\hline 16 & 30.22 & 2.42 & 0.56 & 50.68 \\
\hline 17 & 30.22 & 2.42 & 0.56 & 50.68 \\
\hline 18 & 30.22 & 2.42 & 0.56 & 50.68 \\
\hline 19 & 30.22 & 2.42 & 0.56 & 50.68 \\
\hline 20 & 30.22 & 2.42 & 0.56 & 50.68 \\
\hline
\end{tabular}

Table 2. The qualitative evaluation of images at different iteration level for EZW

\begin{tabular}{|l|l|l|l|l|}
\hline & \multicolumn{4}{l}{ EZW } \\
& \multicolumn{4}{l|}{ Time of execution is $\mathbf{1 7 7 . 5 7 5 9 s}$} \\
\hline Steps & CR & BPP & MSE & PSNR \\
\hline 10 & 0.66 & 0.66 & 0.66 & 0.66 \\
\hline 11 & 1.57 & 1.57 & 1.57 & 1.57 \\
\hline 12 & 3.91 & 3.91 & 3.91 & 3.91 \\
\hline 13 & 9.20 & 9.20 & 9.20 & 9.20 \\
\hline 14 & 18.42 & 18.42 & 18.42 & 18.42 \\
\hline 15 & 31.68 & 31.68 & 31.68 & 31.68 \\
\hline 16 & 41.68 & 41.68 & 41.68 & 41.68 \\
\hline 17 & 41.68 & 41.68 & 41.68 & 41.68 \\
\hline 18 & 41.68 & 41.68 & 41.68 & 41.68 \\
\hline 19 & 41.68 & 41.68 & 41.68 & 41.68 \\
\hline 20 & 41.68 & 41.68 & 41.68 & 41.68 \\
\hline
\end{tabular}

Table 3. The qualitative evaluation of images at different iteration level for STW

\begin{tabular}{|l|l|l|l|l|}
\hline & \multicolumn{4}{l}{$\begin{array}{l}\text { STW } \\
\text { Time of execution is } \mathbf{1 5 0 . 5 7 2 2 s}\end{array}$} \\
\hline Steps & CR & BPP & MSE & PSNR \\
\hline 10 & 0.56 & 0.56 & 0.56 & 0.56 \\
\hline 11 & 1.41 & 1.41 & 1.41 & 1.41 \\
\hline 12 & 3.87 & 3.87 & 3.87 & 3.87 \\
\hline 13 & 9.81 & 9.81 & 9.81 & 9.81 \\
\hline 14 & 20.09 & 20.09 & 20.09 & 20.09 \\
\hline 15 & 35.18 & 35.18 & 35.18 & 35.18 \\
\hline 16 & 46.63 & 46.63 & 46.63 & 46.63 \\
\hline 17 & 46.63 & 46.63 & 46.63 & 46.63 \\
\hline 18 & 46.63 & 46.63 & 46.63 & 46.63 \\
\hline 19 & 46.63 & 46.63 & 46.63 & 46.63 \\
\hline 20 & 46.63 & 46.63 & 46.63 & 46.63 \\
\hline
\end{tabular}

\section{CONCLUSION}

The challenges posed by medical imaging borders on the development of compression algorithms that are nearly lossless for diagnoses as well as support high-compression ratios so as to reduce storage and transmission processes. The outcome of 
this work determine the level of iterations required by the algorithms evaluated to achieve reduced bit-rate for effective saving on storage and achieving fast transmission of a remote diagnosis. The simulation results in the experiments show that the three algorithms appears to give extremely good performance for medical image compression, and may be very useful in practical implementations of Tele-radiology and digital picture archiving and communications (PACS) systems.

\section{REFERENCES}

[1] Salomon, D. 2007. Data Compression: The Complete Reference. 4th ed. London: Springer-Verlag.

[2] Dougherty, G. 2009. Digital Image Processing for Medical Applications. New York, USA: Cambridge University Press.

[3] Solomon, C. and Breckon, T. 2011. Fundamentals of Digital Image Processing: A Practical Approach with Examples in Matlab. Oxford, UK: Wiley-Blackwell.

[4] Lin, C., Lee, M., Pan, S. and Lu, C. 2006. Wavelet compression algorithm applied to abdominal ultrasound images. Australian Institute of Radiography; 53: 11-17.

[5] Neri, E., Caramella, D. and Bartolozzi, C. 2008. Image Processing in Radiology: Current Applications. eds. New York: Springer Berlin Heidelberg.

[6] Gonzalez, R. C., Woods, R. E. and Eddins, S. L. 2004. Digital Image Processing Using Matlab. Upper Saddle River, NJ: Pearson Prentice Hall.

[7] Hwang, W. J., Chine, C. F. and Li, K. J. 2003. Scalable medical data compression and transmission using wavelet transform for telemedicine applications, IEEE Trans. on Info Tech in Biomed; 7: 54-63.

[8] Radha, V., and Pushpalakshmi. 2010. Performance analysis of lossy compression algorithm for medical images. J Global Res Comp Sci; 1: 4.

[9] Sudha, V. K. and Sudhakar, R. 2011. Two dimensional medical image compression techniques-A survey. J ICGST-GVIP.

[10] Meyer-Base, A. 2004. Pattern Recognition for Medical Imaging. San Diego, California, USA: Elsevier Academic Press.
[11] Acharya, T. and Ray A. K. 2005. Image Processing: Principles and Applications. Hoboken, New Jersey: Wiley-Interscience.

[12] Burrus, C., Gopinath, R. and Guo, H. 1998. Introduction to Wavelets and Wavelet Transforms - a Primer. Houston, Texas: Prentice Hall International.

[13] Misiti, M., Misiti, Y., Oppenheim, G. and Poggi, J-M. 1996-1997. Wavelet Toolbox for use with MATLAB ${ }^{\circledR}$. User's Guide Version 2. Natick: The Mathsworks.

[14] Soman, K. P., Ramachandran, K. I. and Resari, N. G. 2004. Insight into Wavelets: From Theory to Practice. India Pvt: Prentice-Hall.

[15] Mallat, S. 1998. Wavelet Tour of Signal Processing. USA: Academic Press.

[16] Sayood, K. 2005. Introduction to Data Compression. 3rd ed. San Francisco, CA: Morgan Kaufmann.

[17] Shapiro, J. M. 1993. Embedded image coding using zerotrees of wavelet coefficients. IEEE Trans Signal Proc; 41: 3445-3462.

[18] Amir, S. and William, A. P. 1996. A new fast/efficient image codec based on set partitioning in hierarchical trees. IEEE Trans Circ \& Syst for Video Tech; 6: 243-250.

[19] Misiti, M., Misiti, Y., Oppenheim, G. and Poggi, J-M. 2007. Wavelets and their Applications. Edited. ISTE USA, Newport Beach

[20] Thyagarajan, K. S. 2011. Still Image and Video Compression with Matlab. New Jersy: John Wiley \& Sons publication.

[21] Chang, S. G, Yu, B. and Vetterli, M. 2000. Adaptive wavelet thresholding for image denoising and compression. IEEE Trans on Image Proc; 9: 1532-1546.

[22] Mousa, A. and Odeh, N. 2012. Optimizing the wavelet parameters to improve image compression. Int $\mathrm{J} \mathrm{Adv}$ in Eng \&Tech; 4: 46-52.

[23] Saffor, A. and Ramli, A. R. 2001. A comparative study of image compression between JPEG and wavelet. Malay J Comp Sci; 14: 39-45.

[24] Kaushik, A and Gupta, M. 2012. Analysis of image compression algorithms. Int J Eng Res \& Appl; 2: 773 779 . 